\title{
Anomalous quantum transport in a thin film
}

\author{
Santanu K. Maiti ${ }^{\dagger, \ddagger}, *$ \\ ${ }^{\dagger}$ Theoretical Condensed Matter Physics Division, Saha Institute of Nuclear Physics, \\ 1/AF, Bidhannagar, Kolkata-700 064, India \\ ${ }^{\ddagger}$ Department of Physics, Narasinha Dutt College, 129, Belilious Road, Howrah-711 101, India
}

\begin{abstract}
We present a numerical study of electron transport in a thin film of varying disorder strength with the distance from its surface. A simple tight-binding model is used to describe the system, in which the film is attached to two metallic electrodes and the coupling of this film to the electrodes is illustrated by the Newns-Anderson chemisorption theory. Quite interestingly we observe that, in the smoothly varying disordered film current amplitude increases with the increase of disorder strength in the strong disorder regime, while it decreases in the weak disorder regime. This behavior is completely opposite from a conventional disordered film, where current amplitude always decreases with the increase of disorder strength.
\end{abstract}

PACS No.: 73.23.-b; 73.63.Rt; 73.21.Ac

Keywords: Thin film; Conductance; Current; Disorder

${ }^{*}$ Corresponding Author: Santanu K. Maiti

Electronic mail: santanu.maiti@saha.ac.in 


\section{Introduction}

Improvements of nanoscience and technology have stimulated us to investigate electrical conduction on mesoscopic/nanoscopic scale in a very tunable environment. The transport properties of quantum systems attached to electrodes have been studied extensively over the last few decades both theoretically as well as experimentally due to their possible technological applications. In 1974, Aviram and Ratner $^{1}$ first developed a theoretical formulation for the description of electron conduction in a molecular electronic device. Later many experiments ${ }^{2-6}$ have been performed in several bridge systems to justify the basic mechanisms underlying the electron transport. From the numerous studies of electron transport, exist in the literature, we can able to understand different features, but yet the complete knowledge of the conduction mechanism in this scale is not well established even today. Many significant factors are there which control the electron transport in a molecular bridge, and all these effects have to be taken into account properly to reveal the transport. For our illustrative purpose, here we mention some of them as follows. In order to reveal the dependence of the molecular structure on the electron transport, Ernzerhof et al. ${ }^{7}$ have performed few model calculations and predicted some interesting results. The molecular coupling ${ }^{8}$ to the side attached electrodes is another important parameter that controls the electron transport in a significant way. The most significant issue is probably the effects of quantum interferences of electron waves in different pathways, and several studies $^{8-16}$ are available in the literature describing these effects. In addition to these, dynamical fluctuations provide an active role in the determination of molecular transport which can be manifested through the measurement of shot noise, a direct consequence of the quantization of charge. This can be used to obtain information on a system which is not directly available through conductance measurements, and is generally more sensitive to the effects of electron-electron correlations than the average conductance. ${ }^{17,18}$

In this article, we concentrate ourselves on a different aspect, related to the effect of disorder, of quantum transport than the above mentioned issues. The characteristic properties of electron transport in conventional disordered systems are well established in the literature. But there are some special type of nano-scale materials, where the charge carriers are scattered mainly from their surface regions ${ }^{19-23}$ and not from the inner core regions. These systems exhibit several peculiar features in electron transport. For example, in a very recent work Yang et $a .^{21}$ have shown a localization to quasi-delocalization transition in edge disordered graphene nanoribbons by varying the strength of edge disorder. Quite similar in behavior has also been observed in a shell-doped nanowire, ${ }^{22}$ where the electron dynamics undergoes a localization to quasi-delocalization transition beyond some critical doping. In the shell-doped nanowire, the dopant atoms are spatially confined within a few atomic layers in the shell region. This is completely opposite to that of a traditional disordered nanowire, where the dopant atoms are distributed uniformly throughout the system. From the numerous studies of electron transport in many such systems, it can be emphasized that the surface reconstructions, ${ }^{24}$ surface scattering ${ }^{25}$ and surface states $^{26}$ may be quite significant to exhibit several diverse transport properties. Motivated with these investigations, here we consider a particular kind of thin film in which disorder strength varies smoothly from layer to layer with the distance from its surface. In this system, our numerical study predicts a strange behavior of electron transport where current amplitude increases with the increase of disorder strength in the strong disorder limit, while it decreases in the limit of weak disorder. On the other hand, for a traditional disordered thin film current amplitude always decreases with the increase of disorder strength. An analytic approach based on the tight-binding model is used to incorporate the electron transport in the film, and we adopt the NewnsAnderson chemisorption model ${ }^{27-29}$ to describe the side attached electrodes and the interaction of these electrodes with the film.

Our organization of this article is as follows. In Section 2, we describe the model and the theoretical formulation for our calculations. Section 3 discusses the significant results, and at the end, we summarize our results in Section 4.

\section{Model and the theoretical description}

The system of our concern is depicted in Fig. 1, where a thin film is attached to two metallic electrodes, viz, source and drain. In this film, disorder strength varies smoothly from the top most disordered layer (solid line) to-wards the bottom layer, keeping the lowest bottom layer (dashed line) as disorder free. The electrodes are symmetrically attached at the two extreme corners of the bottom 
layer. Using the Green's function formalism ${ }^{30}$ and single channel Landauer conductance formula, ${ }^{30}$ we calculate the transmission probability $(T)$, conductance $(g)$ and current $(I)$ through the film.

At low temperature and bias voltage, the conductance $g$ of the film is obtained from the Landauer conductance formula, ${ }^{30}$

$$
g=\frac{2 e^{2}}{h} T
$$

where the transmission probability $T$ can be written in terms of the retarded and advanced Green's functions $\left(G_{F}^{r}\right.$ and $\left.G_{F}^{a}\right)$ of the film as ${ }^{30}$

$$
T=\operatorname{Tr}\left[\Gamma_{S} G_{F}^{r} \Gamma_{D} G_{F}^{a}\right]
$$

The parameters $\Gamma_{S}$ and $\Gamma_{D}$ describe the couplings

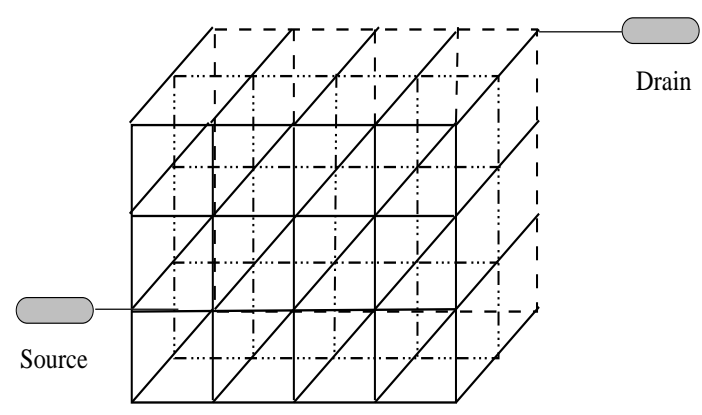

Figure 1: Schematic view of a smoothly varying disordered thin film attached to two metallic electrodes (source and drain). The top most front layer (solid line) is the highest disordered layer and the disorder strength decreases smoothly to-wards the bottom layer keeping the lowest bottom layer (dashed line) as disorder free. Two electrodes are attached at the two extreme corners of the bottom layer.

of the film with the source and drain respectively.

The Green's function of the film becomes,

$$
G_{F}=\left(E-H_{F}-\Sigma_{S}-\Sigma_{D}\right)^{-1}
$$

where $E$ is the energy of the injecting electron and $H_{F}$ corresponds to the Hamiltonian of the film. The parameters $\Sigma_{S}$ and $\Sigma_{D}$ denote the self-energies due to the coupling of the film with the source and drain respectively, and are described by the use of Newns-Anderson chemisorption theory. ${ }^{27-29}$ In the tight-binding framework, the Hamiltonian can be expressed within the non-interacting picture as,

$$
H_{F}=\sum_{i} \epsilon_{i} c_{i}^{\dagger} c_{i}+\sum_{<i j>} t\left(c_{i}^{\dagger} c_{j}+c_{j}^{\dagger} c_{i}\right)
$$

Here $\epsilon_{i}$ gives the on-site energy of an electron at site $i$ and $t$ represents the hopping strength between two nearest-neighbor sites both for the longitudinal and transverse directions of the film. Now to achieve our concerned system, we choose the site energies $\left(\epsilon_{i}\right.$ 's) randomly from a "Box" distribution function such that the top most front layer becomes the highest disordered layer with strength $W$ and the strength of disorder decreases smoothly to-wards the bottom layer as a function of $W /\left(N_{l}-m\right)$, where $N_{l}$ gives the total number of layers and $m$ represents the total number of ordered layers from the bottom side of the film. While, in the conventional disordered thin film all the layers are subjected to the same disorder strength $W$. In our present model, the electrodes are described by the standard tight-binding Hamiltonian, similar to that as prescribed in Eq.(4), and parametrized by constant on-site potential $\epsilon_{0}$ and nearest-neighbor hopping integral $v$.

Assuming the entire voltage is dropped across the film-electrode interfaces, ${ }^{31}$ the current passing through the film, which is regarded as a single electron scattering process between the reservoirs, can be expressed as, ${ }^{30}$

$$
I(V)=\frac{e}{\pi \hbar} \int_{-\infty}^{\infty}\left(f_{S}-f_{D}\right) T(E) d E
$$

where $f_{S(D)}=f\left(E-\mu_{S(D)}\right)$ gives the Fermi distribution function with the electrochemical potential $\mu_{S(D)}=E_{F} \pm e V / 2$. In this article, we focus our study on the determination of the typical current amplitude which is obtained from the relation,

$$
I_{t y p}=\sqrt{<I^{2}>_{W, V}}
$$

where $W$ and $V$ correspond to the impurity strength and the applied bias voltage respectively.

Throughout this presentation, all the results are computed at absolute zero temperature, but they should valid even for finite temperature $(\sim 300 \mathrm{~K})$ as the broadening of the energy levels of the film due to its coupling with the electrodes will be much larger than that of the thermal broadening. ${ }^{30}$ For simplicity, we take the unit $c=e=h=1$ in our present calculations.

\section{Results and discussion}

All the numerical calculations we do here are performed for some particular values of the different parameters, but all the basic features in which we are interested in this particular study remain also invariant for the other parametric values. The values of the different parameters are as follows. The coupling strengths of the film to the electrodes are 
taken as $\tau_{S}=\tau_{D}=1.5$, the nearest-neighbor hopping integral in the film is fixed to $t=1$. The on-site potential and the hopping integral in the electrodes are set as $\epsilon_{0}=0$ and $v=2$ respectively. In addition to these, here we also introduce three other parameters $N_{x}, N_{y}$ and $N_{z}$ to specify the system size of the thin film, where they correspond to the total number of lattice sites along the $x, y$ and $z$ directions of the film respectively. In our numerical calculations, the typical current amplitude $\left(I_{t y p}\right)$ is determined by taking the average over the disordered configurations and bias voltages (see Eq.(6) ). Since in this particular model the site energies are chosen randomly, we compute $I_{t y p}$ by taking the average over a large number (60) of disordered configurations in each case to get much accurate result. On the other hand, for the averaging over the bias voltage $V$, we set the range of it from -10 to 10 . In this presentation, we focus only on the systems with small sizes since all the qualitative behaviors remain also invariant even for the large systems.

Figure 2 illustrates the variation of the typical current amplitude $\left(I_{t y p}\right)$ as a function of the disorder $(W)$ for some thin films with $N_{x}=10, N_{y}=8$ and $N_{z}=5$. Here we set $m=1$, i.e., only the lowest bottom layer is free from any disorder for these films. The solid and dotted curves correspond to the results of the smoothly varying and complete disordered thin films respectively. A remarkably different behavior is observed for the smoothly varying disordered film compared to the film with complete disorder. In the later system, it is observed that $I_{t y p}$ decreases rapidly with $W$ and eventually it drops to zero for the higher value of $W$. This reduction of the current is due to the fact that the eigenstates become more localized ${ }^{32}$ with the increase of disorder, and it is well established from the theory of Anderson localization. ${ }^{33}$ The appreciable change in the variation of the typical current amplitude takes place only for the unconventional disordered film. In this case, the current amplitude decreases initially with $W$ and after reaching to a minimum at $W=W_{c}$ (say), it again increases. Thus the anomalous behavior is observed beyond the critical disorder strength $W_{c}$, and we are interested particularly in this regime where $W>W_{c}$. In order to illustrate this peculiar behavior, we consider the smoothly varying disordered film as a coupled system combining two sub-systems. The coupling exists between the lowest bottom ordered layer and the other disordered layers. Thus the system can be treated, in other way, as a coupled order-disorder separated thin film. For this coupled system we can write the Schrödinger equations as:
$\left(H_{0}-H_{1}\right) \psi_{0}=E \psi_{0}$ and $\left(H_{d}-H_{2}\right) \psi_{d}=E \psi_{d}$ Here $H_{0}$ and $H_{d}$ represent the sub-Hamiltonians of the ordered and disordered regions of the film respectively, and $\psi_{0}$ and $\psi_{d}$ are the corresponding eigenfunctions. The terms $H_{1}$ and $H_{2}$ in the above two expressions are the most significant and they



Figure 2: $I_{t y p}$ vs. $W$ for some thin films with $N_{x}=$ $10, N_{y}=8$ and $N_{z}=5$. Here we set $m=1$. The solid and dotted curves correspond to the smoothly varying and complete disordered films respectively.

can be expressed as: $H_{1}=H_{o d}\left(H_{d}-E\right)^{-1} H_{d o}$ and $H_{2}=H_{d o}\left(H_{o}-E\right)^{-1} H_{o d} . H_{o d}$ and $H_{d o}$ correspond to the coupling between the ordered region and the disordered region. From these mathematical expressions, the anomalous behavior of the electron transport in the film can be described clearly. In the absence of any interaction between the ordered and disordered regions, we can assume the full system as a simple combination of two independent subsystems. Therefore, we get all the extended states in the ordered region, while the localized states are obtained in the disordered region. In this situation, the motion of an electron in any one region is not affected by the other. But for the coupled system, the motion of the electron is no more independent, and we have to take the combined effects coming from both the two regions. With the increase of disorder, the scattering effect becomes dominated more, and thus the reduction of the current is expected. This scattering is due to the existence of the localized eigenstates in the disordered regions. Therefore, in the case of strong coupling between the two subsystems, the motion of the electron in the ordered region is significantly influenced by the disordered regions. Now the degree of this coupling between the two sub-systems solely depends on the two parameters $H_{1}$ and $H_{2}$, those are expressed earlier. In the limit of weak disorder, the scattering effect from both the two regions is quite significant since then the terms $H_{1}$ and $H_{2}$ have reasonably high values. 
With the increase of disorder, $H_{1}$ decreases gradually and for a very large value of $W$ it becomes very small. Hence the term $\left(H_{0}-H_{1}\right)$ effectively goes to $H_{0}$ in the limit $W \rightarrow 0$, which indicates that the ordered region becomes decoupled from the disordered one. Therefore, in the higher disorder regime



Figure 3: $I_{t y p}$ vs. $W$ for some thin films with $N_{x}=$ $12, N_{y}=10$ and $N_{z}=6$. Here we set $m=2$. The solid and dotted curves correspond to the identical meaning as in Fig. 2 .

the scattering effect becomes less significant from the ordered region, and it decreases with $W$. For the low regime of $W$, the eigenstates of both the two effective Hamiltonians, $\left(H_{0}-H_{1}\right)$ and $\left(H_{d}-H_{2}\right)$, are localized. With the increase of $W, H_{1}$ gradually decreases, resulting in much weaker localization in the states of $\left(H_{0}-H_{1}\right)$, while the states of $\left(H_{d}-H_{2}\right)$ become more localized. At a critical value of $W=W_{c}$ (say) ( $\simeq$ band width of $H_{0}$ ), we get a separation between the much weaker localized states and the strongly localized states. Beyond this value, the weaker localized states become more extended and the strongly localized states become more localized with the increase of $W$. In this situation, the current is obtained mainly from these nearly extended states which provide the larger current with $W$ in the higher disorder regime.

To illustrate the size dependence of the film on the electron transport, in Fig. 3 we plot the variation of the typical current amplitude for some thin films with $N_{x}=12, N_{y}=10$ and $N_{z}=6$. For these films we take $m=2$, i.e., two layers from the bottom side are free from any disorder. The solid and dotted curves correspond to the identical meaning as in Fig. 2. For both the unconventional and traditional disordered films, we get almost the similar behavior of the current as presented in Fig. 2. This study shows that the typical current amplitude strongly depends on the finite size of the thin film.

\section{Concluding Remarks}

To summarize, we have done a numerical study to show the anomalous behavior of electron transport in a unconventional disordered thin film where the disorder strength varies smoothly from its surface. A simple tight-binding model has been used to describe the system, where the coupling of the film to the electrodes has been described by the Newns-Anderson chemisorption theory. We have calculated the typical current amplitudes by using the Green's function formalism, and our results have shown a remarkably different behavior for the unconventional disordered film compared to the traditional disordered film. In the smoothly varying disordered film, the typical current amplitude decreases with $W$ in the weak disorder regime $\left(W<W_{c}\right)$, while it increases in the strong disorder regime $\left(W>W_{c}\right)$. On the other hand for the conventional disordered film, the current amplitude always decreases with disorder. In this present investigations, we have also studied the finite size effects of the film and our numerical results have shown that the typical current amplitude strongly depends on the size of the film. Quite similar feature of anomalous quantum transport can also be observed in some other lower dimensional systems like, edge disordered graphene sheets of single-atom-thick, finite width rings with surface disorder, nanowires, etc. Our study has revealed that the carrier transport in an order-disorder separated mesoscopic device may be tailored to desired properties through doping for different applications.

\section{References}

[1] A. Aviram and M. Ratner, Chem. Phys. Lett. 29, 277 (1974).

[2] T. Dadosh, Y. Gordin, R. Krahne, I. Khivrich, D. Mahalu, V. Frydman, J. Sperling, A. Yacoby, and I. Bar-Joseph, Nature 436, 677 (2005).

[3] R. M. Metzger et al., J. Am. Chem. Soc. 119, 10455 (1997).

[4] C. M. Fischer, M. Burghard, S. Roth, and K. V. Klitzing, Appl. Phys. Lett. 66, 3331 (1995).

[5] J. Chen, M. A. Reed, A. M. Rawlett, and J. M. Tour, Science 286, 1550 (1999).

[6] M. A. Reed, C. Zhou, C. J. Muller, T. P. Burgin, and J. M. Tour, Science 278, 252 (1997). 
[7] M. Ernzerhof, M. Zhuang, and P. Rocheleau, J. Chem. Phys. 123, 134704 (2005).

[8] R. Baer and D. Neuhauser, Chem. Phys. 281, 353 (2002).

[9] K. Tagami, L. Wang, and M. Tsukada, Nano Lett. 4, 209 (2004).

[10] M. Magoga and C. Joachim, Phys. Rev. B 59, 16011 (1999).

[11] J.-P. Launay and C. D. Coudret, in: A. Aviram and M. A. Ratner (Eds.), Molecular Electron$i c s$, New York Academy of Sciences, New York, (1998).

[12] R. Baer and D. Neuhauser, J. Am. Chem. Soc. 124, 4200 (2002).

[13] D. Walter, D. Neuhauser, and R. Baer, Chem. Phys. 299, 139 (2004).

[14] K. Walczak, Cent. Eur. J. Chem. 2, 524 (2004).

[15] R. H. Goldsmith, M. R. Wasielewski, and M. A. Ratner, J. Phys. Chem. B 110, 20258 (2006).

[16] M. Ernzerhof, H. Bahmann, F. Goyer, M. Zhuang, and P. Rocheleau, J. Chem. Theory Comput. 2, 1291 (2006).

[17] Y. M. Blanter and M. Buttiker, Phys. Rep. 336, 1 (2000).

[18] K. Walczak, Phys. Stat. Sol. (b) 241, 2555 (2004).

[19] L. P. Kouwenhoven, C. M. Marcus, P. L. McEuen, S. Tarucha, R. M. Westervelt, and N. S. Wingreen, in Mesoscopic Electron Transport: Proc. NATO Advanced Study Institutes (NATO Advanced Study Institute, Series E: Applied Sciences) 345, (1997).

[20] H. B. Chen and J. W. Ding, Physica B 403, 2015 (2008).

[21] C. Y. Yang, J. W. Ding, and N. Xu, Physica B 394, 69 (2007).

[22] J. X. Zhong and G. M. Stocks, Nano. Lett. 6, 128 (2006).

[23] J. X. Zhong and G. M. Stocks, Phys. Rev. B 75, 033410 (2007).

[24] R. Rurali and N. Lorente, Phys. Rev. Lett. 94, 026805 (2005).
[25] Y. Cui, X. F. Duan, J. T. Hu, and C. M. Lieber, J. Phys. Chem. B 104, 5213 (2000).

[26] J. Y. Yu, S. W. Chung, and J. R. Heath, J. Phys. Chem. B 104, 11864 (2000).

[27] V. Mujica, M. Kemp, and M. A. Ratner, J. Chem. Phys. 101, 6849 (1994).

[28] V. Mujica, M. Kemp, A. E. Roitberg, and M. A. Ratner, J. Chem. Phys. 104, 7296 (1996).

[29] D. M. Newns, Phys. Rev. 178, 1123 (1969).

[30] S. Datta, Electronic transport in mesoscopic systems, Cambridge University Press, Cambridge (1997).

[31] W. Tian, S. Datta, S. Hong, R. Reifenberger, J. I. Henderson, and C. I. Kubiak, J. Chem. Phys. 109, 2874 (1998).

[32] P. A. Lee and T. V. Ramakrishnan, Rev. Mod. Phys. 57, 287 (1985).

[33] P. W. Anderson, Phys. Rev. 109, 1492 (1958). 